\title{
ASSESSMENT OF PLANT DIVERSITY AND PRIORITIZATION OF COMMUNITIES FOR CONSERVATION IN MORNAULA RESERVE FOREST
}

\author{
S. PANT - S.S. SAMANT* \\ G.B. Pant Institute of Himalayan Environment and Development, Himachal Unit, \\ Mohal-Kullu 175 126, Himachal Pradesh, India \\ (phone: +91-1902-225329 Ext. 21 (O); fax: +91-1902-226347) \\ e-mail:samantss2@rediffmail.com;samant62@yahoo.com
}

(Received $4^{\text {th }}$ July2006; accepted $16^{\text {th }}$ August 2007)

\begin{abstract}
Assessment of plant diversity of the Reserve Forests of the west Himalaya and prioritization of communities for conservation have not been given much attention. Therefore, the study has been conducted in a biodiversity rich Mornaula Reserve Forest between $1500-2200 \mathrm{~m}$ to analyse the structure, composition of the forest communities including richness of economically important, native, endemic and rare-endangered species, and prioritize communities for conservation. A total of 123 sites were sampled. For each site, habitat characteristics, altitude and dominant species have been given. From the sampled sites, 289 species ( 37 trees; 37 shrubs; and 215 herbs) and 31 forest communities have been recorded. The density of trees ranged from 340-2438 $\mathrm{Ind} \mathrm{ha}^{-1}$ and TBA from 19.52-234.31 Ind $\mathrm{m}^{2}$. The densities of saplings ranged from 340.00-2277.00 Ind ha ${ }^{-1}$ and seedlings 266.00-1571.00 Ind ha ${ }^{-1}$; shrubs 357-1156 Ind $\mathrm{ha}^{-1}$ and herbs 21.73-431.04 Ind $\mathrm{m}^{-2}$. The richness of the trees ranged from 3-27, shrubs, 8-36, herbs, 17-145, seedlings, 3-22, and saplings, 2-21. Species diversity for trees ranged from 0.99-2.93, seedlings, 0.86-2.65, saplings, $0.44-2.78$, shrubs, $1.94-4.43$ and herbs, $1.42-4.66$. These recorded values were almost comparable with the studies conducted in sub-tropical, temperate and sub-alpine regions of the west Himalaya. In some cases the values were slightly higher than the reported values. The communities have been prioritized for conservation based on the species richness, nativity, endemism, economically important and rare-endangered species. Among, all the prioritized communities, Rhododendron arboreum community supports maximum species including native, endemic, economically important and rareendangered species. In view of the high socio-economic and conservation values of the identified communities, monitoring of these communities at least for a period of five years and development of appropriate strategy and action plan for the conservation and management have been suggested.
\end{abstract}

Keywords: Reserve Forest, communities, diversity, native, rare-endangered, socioeconomic, prioritization, conservation

\section{Introduction}

The Indian Himalayan Region (IHR) is very well known throughout the globe due to its representative, unique, natural, and socio-economically important flora and fauna [1]. Due to this peculiar feature, the eastern Himalaya has been identifies one of the biodiversity Hot Spots [2]. This rich biodiversity is being utilized by the inhabitants of the region for medicine, as wild edible (food), fodder, fuel, timber, in making agriculture tools, religious and various other purposes $[3,4,1]$. With the increasing human population, the demand of the economically important biodiversity is increasing fast. Collection of fodder and fuel species from the forests has been identified one of the chronic problems in the IHR for the degradation of forest [5]. The anthropogenic pressures including heavy grazing coupled with the natural calamities have lead the degradation of natural habitats of many species to a great extent. Such practices are discoursing the moisture loving species and promoting the hardy and spiny species having least value for the society. This loss of biodiversity and changing pattern of 
vegetation has necessitated assess the biodiversity of the region and prioritize habitats, communities and species for conservation.

In general, structural and functional diversity of the some parts of the IHR have been evaluated by various workers [6-33]. However, the protected areas of the IHR including Reserve Forests have been very poorly evaluated for the structural and functional diversity [34] except a few studies carried out in Nanda Devi Biosphere Reserve (NDBR) and Askot Wildlife Sanctuary (AWLS) [35-39]. Further, studies integrating compositional, structural and functional diversity, native, endemic, economically important and rare-endangered species, and prioritization of community for conservation have been attempted in a few protected areas [37, 38]. Therefore, the attempt has been made to; (i) study the site/habitat characteristics; (ii) assess the diversity and distribution pattern of the species; (iii) delineate forest communities; (iv) study the distribution pattern of economically important, native, endemic and rareendangered species within the identified communities; and (v) prioritize communities for conservation.

\section{Materials and methods}

\section{Identification and selection of transects, sites and habitats}

Four transacts i.e., (i) Harinagar to Nartola; (ii) Bercheula-Lohanigaon-Mornaula; (iii) Khakar-Bheutania-Tarani; (iv) Dole-Damar-Mornaula were selected along the trails of the villages on account of typical topography and inaccessibility of the area. The sites were selected on each and every accessible aspect along transacts between 1500-2200m. The habitats were identified on the basis of physical characters and dominance of the vegetation. Sites having closed canopy with high percent of humus and moisture were considered as moist habitats whereas low percent of the same as dry habitats. The sites having $>50 \%$ boulders of the ground cover had been considered as bouldary habitat, and the sites facing high anthropogenic pressures considered as degraded habitats.

\section{Survey, sampling, identification and analysis of data}

The field surveys and samplings were conducted between 2002 and 2004 within the selected sites along the transacts. In each site, a plot of 50x50m was laid. Trees, saplings and seedlings were sampled by randomly placed $10,10 \times 10 \mathrm{~m}$ quadrates; shrubs by 10 , $5 \times 5 \mathrm{~m}$ quadrates; herbs by $20,1 \times 1 \mathrm{~m}$ quadrates in each plot. The size and number of quadrates was determined following [40]. For the collection of data from these quadrates standard ecological methods [41, 42, 40, 43, 35, 39, 38] were followed. From each site, samples of each species were collected and identified in the Institute with the help of florulas and research papers [44, 45, 46, 29].

For trees, basal area and Importance Value Index (IVI) have also been computed. IVI has been calculated as the sum of relative frequency, relative density and relative basal area. The abundance data of different sites were pooled to get community averages in terms of density, total basal area and IVI. Communities were identified based on the IVI. A species contributing $50 \%$ or $>50 \%$ of the total IVI in a particular site/habitat is considered as a single species dominated community, $<50 \%$ of the total IVI is named as a mixed community. 


\section{Species diversity $\left(H^{\prime}\right)$ and Concentration of dominance $(C d)$}

Species richness is the total number of species in a particular community. Species diversity was determined by Shannon Wiener's information statistic (H') [47] and concentration of dominance by [48]

\section{Identification of native, endemic and rare-endangered species}

Native species were identified following [49, 1, 50, 28, 29]. The species having their origin in the Himalayan region have been considered as natives. Endemism is based on the distribution range of the species $[51,1,36]$. The species restricted to the IHR have been considered as endemic whereas those with extended distribution to neighbouring countries/States as near endemic. Rare-Endangered species has been identified based on habitat specificity, population size, distribution range and anthropogenic pressures [52, $53,36]$.

\section{Prioritization of communities}

Based on the occurrence of number of species (species richness), economically important, native, endemic and rare-endangered species, within the communities, prioritization of the communities for conservation has been done.

\section{Results and discussion}

\section{Site and habitat characteristics}

Site/habitat characteristic, dominant species, altitude, slope and aspect of all four transects are presented (Table 1). The altitude ranged between $1500-2200 \mathrm{~m}$ and majority of the study sites fall in northeast aspect. In all five habitats i.e., shady moist, dry, riverine, bouldary, and degraded were represented in the area (Table 1).

\section{Community diversity and distribution pattern}

Thirty-one forest communities have been identified between $1500-2200 \mathrm{~m}$ in the Mornaula Reserve Forest (MRF). The community types, altitudinal distribution, sites and habitat representation and major tree associates are presented in (Table 2). Rhododendron arboreum community represented maximum sites (26), followed by Quercus leucotrichophora (18 sites), and Pinus roxburghii (16 sites), and the remaining communities showed less representation of sites. Among the communities Rhododendron arboreum, Quercus leucotrichophora, Pinus roxburghii, Quercus floribunda, Cupressus torulosa, and Quercus leucotrichophora-Rhododendron arboreum mixed, showed comparatively wide altitudinal range of distribution. 
Table 1. Physical characteristics of sites in the MRF

Transact 1: Nartola to Harinagar

\begin{tabular}{cccccc}
\hline S.No. & $\begin{array}{c}\text { Altitude } \\
(\mathbf{m})\end{array}$ & $\begin{array}{c}\text { Slope } \\
(\mathbf{(})\end{array}$ & $\begin{array}{c}\text { Habitat } \\
(\mathbf{s})\end{array}$ & Aspect & Dominant Species \\
\hline 1 & 2130 & 10 & $\mathrm{D}$ & $\mathrm{E}$ & Quercus floribunda \& Quercus leucotrichophora \\
2 & 2130 & 20 & $\mathrm{~B}$ & $\mathrm{E}$ & Quercus leucotrichophora \& Quercus floribunda \\
3 & 2130 & 5 & $\mathrm{~B}$ & $\mathrm{SW}$ & Quercus floribunda \& Quercus leucotrichophora \\
4 & 2130 & 15 & $\mathrm{~B}$ & $\mathrm{~S}$ & Rhododendron arboreum \& Quercus floribunda \\
5 & 2130 & 20 & $\mathrm{~B}$ & $\mathrm{~N}$ & Rhododendron arboreum \& Quercus floribunda \\
6 & 2130 & 25 & $\mathrm{~B}$ & $\mathrm{NE}$ & Quercus floribunda \& Betula alnoides \\
7 & 2120 & 20 & $\mathrm{~B}$ & $\mathrm{SE}$ & Betula alnoides, Quercus floribunda, Lyonia ovalifolia \\
8 & 2120 & 25 & $\mathrm{~B}$ & $\mathrm{SE}$ & Rhododendron arboreum, Quercus floribunda, Alnus \\
& & & & & nepalensis \\
9 & 2120 & 45 & $\mathrm{~B}$ & $\mathrm{NE}$ & Quercus leucotrichophora, Rhododendron arboreum, \\
& & & & & Betula alnoides \\
10 & 2125 & 25 & $\mathrm{D}$ & $\mathrm{S}$ & Quercus leucotrichophora \\
11 & 2120 & 15 & $\mathrm{~B}$ & $\mathrm{NE}$ & Quercus leucotrichophora \& Quercus floribunda \\
12 & 2115 & 10 & $\mathrm{C}$ & $\mathrm{NE}$ & Quercus floribunda \\
13 & 2115 & 30 & $\mathrm{~B}$ & $\mathrm{NE}$ & Quercus floribunda \\
14 & 2120 & 30 & $\mathrm{~B}$ & $\mathrm{NW}$ & Rhododendron arboreum \\
15 & 2125 & 15 & $\mathrm{~B}$ & $\mathrm{SE}$ & Rhododendron arboreum \\
16 & 2125 & 20 & $\mathrm{~B}$ & $\mathrm{E}$ & Quercus leucotrichophora \& Rhododendron arboreum \\
17 & 2125 & 35 & $\mathrm{~B}$ & $\mathrm{SE}$ & Rhododendron arboreum \& Quercus leucotrichophora \\
18 & 2070 & 45 & $\mathrm{~B}$ & $\mathrm{NE}$ & Abies pindrow \\
19 & 2070 & 40 & $\mathrm{~B}$ & $\mathrm{NW}$ & Abies pindrow \& Quercus leucotrichophora \\
20 & 2125 & 40 & $\mathrm{~B}$ & $\mathrm{NW}$ & Quercus leucotrichophora \& Rhododendron arboreum \\
21 & 2125 & 40 & $\mathrm{~B}$ & $\mathrm{E}$ & Rhododendron arboreum \& Quercus leucotrichophora \\
22 & 2120 & 35 & $\mathrm{~B}$ & $\mathrm{SW}$ & Abies pindrow \\
23 & 2125 & 5 & $\mathrm{D}$ & NW & Rhododendron arboreum \& Quercus leucotrichophora \\
24 & 2120 & 20 & $\mathrm{C}$ & $\mathrm{SE}$ & Persea duthiei \& Rhododendron arboreum \\
\hline
\end{tabular}

Transact 2: Bercheula - Lohanigaon-Mornaula

\begin{tabular}{|c|c|c|c|c|c|}
\hline S.No. & $\begin{array}{c}\text { Altitude } \\
(\mathrm{m})\end{array}$ & $\begin{array}{c}\text { Slope } \\
\left({ }^{\circ}\right)\end{array}$ & $\begin{array}{c}\text { Habitat } \\
\text { (s) }\end{array}$ & Aspect & Dominant Species \\
\hline 1 & 1900 & 40 & $\mathrm{~A}$ & $\mathrm{~S}$ & Pinus roxburghii \\
\hline 2 & 1860 & 10 & $\mathrm{C}$ & $\mathrm{E}$ & Rhododendron arboreum, Daphniphyllum himalayense \\
\hline 3 & 1870 & 40 & $\mathrm{~B}$ & $\mathrm{~N}$ & $\begin{array}{l}\text { Rhododendron arboreum, Aesculus indica, Quercus } \\
\text { floribunda }\end{array}$ \\
\hline 4 & 1870 & 40 & A & $\mathrm{NE}$ & Pinus roxburghii \\
\hline 5 & 1890 & 20 & $\mathrm{C}$ & $\mathrm{NE}$ & Rhododendron arboreum \\
\hline 6 & 1900 & 40 & A & $\mathrm{NE}$ & Pinus roxburghii \\
\hline 7 & 1890 & 20 & $\mathrm{C}$ & NW & Alnus nepalensis \\
\hline 8 & 1970 & 45 & B & $\mathrm{NE}$ & Rhododendron arboreum \\
\hline 9 & 1970 & 40 & B & $\mathrm{NE}$ & Rhododendron arboreum \\
\hline 10 & 1960 & 40 & $\mathrm{D}$ & NW & Pinus roxburghii \\
\hline 11 & 1960 & 40 & B & $\mathrm{W}$ & Cedrus deodara \\
\hline 12 & 1950 & 50 & $\mathrm{~B}$ & $\mathrm{E}$ & Cupressus torulosa \\
\hline 13 & 2050 & 20 & $\mathrm{~B}$ & $\mathrm{~S}$ & Cedrus deodara \\
\hline 14 & 2060 & 30 & $\mathrm{C}$ & SE & Rhododendron arboreum \\
\hline 15 & 2040 & 20 & $\mathrm{C}$ & SE & Alnus nepalensis \\
\hline 16 & 2070 & 40 & $\mathrm{~A}$ & $\mathrm{~S}$ & Quercus leucotrichophora \\
\hline 17 & 2090 & 35 & A & $\mathrm{S}$ & Pinus roxburghii \\
\hline 18 & 2095 & 45 & $\mathrm{~B}$ & NW & Rhododendron arboreum \\
\hline
\end{tabular}


Transact 2: Bercheula - Lohanigaon-Mornaula

\begin{tabular}{|c|c|c|c|c|c|}
\hline S.No. & $\begin{array}{l}\text { Altitude } \\
(\mathrm{m})\end{array}$ & $\begin{array}{c}\text { Slope } \\
\left({ }^{\circ}\right)\end{array}$ & $\begin{array}{c}\text { Habitat } \\
\text { (s) }\end{array}$ & Aspect & Dominant Species \\
\hline 19 & 2090 & 40 & $\mathrm{E}$ & SW & Pinus roxburghii \\
\hline 20 & 2090 & 40 & A & W & Pinus roxburghii \\
\hline 21 & 2100 & 20 & B & W & Rhododendron arboreum \\
\hline 22 & 2090 & 35 & B & $\mathrm{N}$ & Rhododendron arboreum \& Myrica esculenta \\
\hline 23 & 2095 & 35 & B & SE & Cupressus torulosa \\
\hline 24 & 2070 & 35 & $\mathrm{C}$ & NW & Rhododendron arboreum \& Alnus nepalensis \\
\hline 25 & 2070 & 35 & A & W & Pinus roxburghii \\
\hline 26 & 2095 & 45 & B & E & Myrica esculenta \\
\hline 27 & 2095 & 25 & B & SW & Cupressus torulosa \\
\hline 28 & 2095 & 35 & B & SE & Myrica esculenta \\
\hline 29 & 2080 & 45 & B & SW & Cupressus torulosa \\
\hline 30 & 2105 & 60 & B & $\mathrm{N}$ & Rhododendron arboreum \\
\hline 31 & 2105 & 50 & B & NW & Rhododendron arboreum \\
\hline 32 & 2110 & 40 & B & $\mathrm{NE}$ & Rhododendron arboreum \\
\hline 33 & 2110 & 25 & B & $\mathrm{W}$ & Rhododendron arboreum \\
\hline 34 & 2120 & 35 & $\mathrm{C}$ & SE & Persea duthiei \& Litsea umbrosa \\
\hline 35 & 2115 & 20 & $\mathrm{C}$ & $\mathrm{NE}$ & Betula alnoides \& Rhododendron arboreum \\
\hline 36 & 2115 & 20 & B & $\mathrm{N}$ & Rhododendron arboreum \& Lyonia ovalifolia \\
\hline 37 & 2105 & 25 & B & $\mathrm{NE}$ & Betula alnoides \& Rhododendron arboreum \\
\hline 38 & 2105 & 20 & B & $\mathrm{NE}$ & Rhododendron arboreum \\
\hline 39 & 2100 & 5 & $\mathrm{C}$ & SE & Acer cappadocicum \& Persea duthiei \\
\hline 40 & 1990 & 10 & $\mathrm{C}$ & SE & Quercus floribunda \\
\hline 41 & 1985 & 40 & B & $\mathrm{NE}$ & Quercus leucotrichophora \\
\hline 42 & 1990 & 15 & B & $\mathrm{S}$ & Rhododendron arboreum \\
\hline 43 & 2105 & 15 & B & $\mathrm{E}$ & Quercus floribunda \& Quercus leucotrichophora \\
\hline 44 & 2110 & 15 & $\mathrm{D}$ & SW & Quercus floribunda \\
\hline
\end{tabular}

Transact 3: Khakar-Bheutania-Tarani

\begin{tabular}{|c|c|c|c|c|c|}
\hline S.No. & $\begin{array}{l}\text { Altitude } \\
\text { (m) }\end{array}$ & $\begin{array}{c}\text { Slope } \\
\left({ }^{\circ}\right)\end{array}$ & $\begin{array}{c}\text { Habitat } \\
\text { (s) }\end{array}$ & Aspect & Dominant Species \\
\hline 1 & 2010 & 15 & $\mathrm{C}$ & $\mathrm{E}$ & Quercus floribunda \\
\hline 2 & 2020 & 45 & A & $\mathrm{S}$ & Quercus floribunda \\
\hline 3 & 2030 & 35 & A & $\mathrm{E}$ & Quercus floribunda \& Quercus leucotrichophora \\
\hline 4 & 2060 & 45 & $\mathrm{~B}$ & $\mathrm{NE}$ & Rhododendron arboreum \\
\hline 5 & 2080 & 35 & $\mathrm{C}$ & $\mathrm{E}$ & Rhododendron arboreum \& Betula alnoides \\
\hline 6 & 2100 & 35 & A & SE & Quercus floribunda \\
\hline 7 & 2100 & 50 & B & $\mathrm{NE}$ & Rhododendron arboreum \\
\hline 8 & 2075 & 35 & $\mathrm{C}$ & $\mathrm{NE}$ & Rhododendron arboreum \\
\hline 9 & 2100 & 25 & $\mathrm{D}$ & $\mathrm{N}$ & Rhododendron arboreum \\
\hline 10 & 2100 & 45 & $\mathrm{C}$ & $\mathrm{NE}$ & Rhododendron arboreum \\
\hline 11 & 2100 & 40 & B & NW & Rhododendron arboreum \\
\hline 12 & 2110 & 25 & B & NW & Quercus leucotrichophora \\
\hline 13 & 2115 & 25 & $\mathrm{~B}$ & NW & Quercus leucotrichophora \\
\hline 14 & 2110 & 5 & B & $\mathrm{E}$ & Persea duthiei \& Rhododendron arboreum \\
\hline 15 & 2015 & 50 & A & $\mathrm{S}$ & Rhododendron arboreum \& Quercus floribunda \\
\hline 16 & 2010 & 40 & $\mathrm{C}$ & $\mathrm{N}$ & Rhododendron arboreum \\
\hline 17 & 2030 & 40 & A & $\mathrm{E}$ & Pinus roxburghii \\
\hline 18 & 1940 & 30 & B & $\mathrm{N}$ & Quercus floribunda \\
\hline 19 & 1960 & 45 & $\mathrm{D}$ & $\mathrm{N}$ & Pinus roxburghii \\
\hline 20 & 1960 & 50 & $\mathrm{D}$ & SW & Pinus roxburghii \\
\hline 21 & 1940 & 35 & $\mathrm{D}$ & $\mathrm{E}$ & Quercus floribunda \\
\hline
\end{tabular}


Transact 3: Khakar-Bheutania-Tarani

\begin{tabular}{cccccc}
\hline S.No. & $\begin{array}{c}\text { Altitude } \\
(\mathbf{m})\end{array}$ & $\begin{array}{c}\text { Slope } \\
(\mathbf{(})\end{array}$ & $\begin{array}{c}\text { Habitat } \\
(\mathbf{s})\end{array}$ & Aspect & Dominant Species \\
\hline 22 & 1960 & 55 & $\mathrm{~A}$ & $\mathrm{SW}$ & Pinus roxburghii \\
23 & 1950 & 50 & $\mathrm{~A}$ & $\mathrm{E}$ & Pinus roxburghii \\
24 & 1940 & 10 & $\mathrm{C}$ & $\mathrm{SE}$ & Daphniphyllum himalayense \\
25 & 1650 & 50 & $\mathrm{~B}$ & $\mathrm{~N}$ & Quercus leucotrichophora \\
26 & 1650 & 70 & $\mathrm{~B}$ & $\mathrm{SW}$ & Quercus leucotrichophora \\
27 & 1720 & 40 & $\mathrm{~A}$ & $\mathrm{SW}$ & Quercus leucotrichophora \\
28 & 1790 & 45 & $\mathrm{~B}$ & $\mathrm{NW}$ & Quercus leucotrichophora \\
29 & 1790 & 60 & $\mathrm{~B}$ & $\mathrm{SW}$ & Myrica esculenta \& Quercus leucotrichophora \\
30 & 1790 & 30 & $\mathrm{C}$ & $\mathrm{NE}$ & Quercus leucotrichophora \& Rhododendron arboreum \\
31 & 1800 & 65 & $\mathrm{~B}$ & $\mathrm{NW}$ & Quercus leucotrichophora \\
32 & 1840 & 50 & $\mathrm{~B}$ & $\mathrm{~W}$ & Quercus leucotrichophora \\
33 & 1860 & 70 & $\mathrm{~A}$ & $\mathrm{~S}$ & Pinus roxburghii \\
\hline
\end{tabular}

Transact 4: Dol-Damar-Mornaula

\begin{tabular}{|c|c|c|c|c|c|}
\hline S.No. & $\begin{array}{c}\text { Altitude } \\
\text { (m) }\end{array}$ & $\begin{array}{l}\text { Slope } \\
\left({ }^{\circ}\right)\end{array}$ & $\begin{array}{l}\text { Habitat } \\
\text { (s) }\end{array}$ & Aspect & Dominant Species \\
\hline 1 & 1960 & 15 & A & E & Quercus leucotrichophora \\
\hline 2 & 1950 & 50 & B & $\mathrm{N}$ & Cedrus deodara \\
\hline 3 & 1910 & 15 & $\mathrm{C}$ & $\mathrm{N}$ & Cedrus deodara \\
\hline 4 & 2030 & 30 & A & $\mathrm{S}$ & Pinus roxburghii \\
\hline 5 & 2030 & 65 & B & SE & Cupressus torulosa \\
\hline 6 & 2025 & 40 & $\mathrm{C}$ & SE & Quercus leucotrichophora \\
\hline 7 & 2025 & 35 & A & SE & Quercus leucotrichophora \\
\hline 8 & 2070 & 50 & B & $\mathrm{S}$ & Quercus leucotrichophora \\
\hline 9 & 2060 & 70 & A & SW & Quercus leucotrichophora \\
\hline 10 & 2080 & 50 & B & SW & Rhododendron arboreum \\
\hline 11 & 2080 & 50 & B & NW & Myrica esculenta \\
\hline 12 & 2085 & 45 & A & SW & Pinus roxburghii \\
\hline 13 & 2100 & 40 & B & $\mathrm{N}$ & Rhododendron arboreum \\
\hline 14 & 2100 & 10 & B & $\mathrm{N}$ & Quercus leucotrichophora \& Rhododendron arboreum \\
\hline 15 & 2105 & 15 & $\mathrm{D}$ & NE & Quercus leucotrichophora \& Rhododendron arboreum \\
\hline 16 & 2110 & 30 & B & $\mathrm{NE}$ & Rhododendron arboreum \\
\hline 17 & 2120 & 15 & $\mathrm{C}$ & NE & Rhododendron arboreum \\
\hline 18 & 2110 & 20 & $\mathrm{C}$ & $\mathrm{NE}$ & Persea odoratissima \\
\hline 19 & 2110 & 15 & B & $\mathrm{E}$ & Litsea umbrosa \& Rhododendron arboreum \\
\hline 20 & 2110 & 15 & B & E & Rhododendron arboreum \& Quercus floribunda \\
\hline 21 & 2110 & 10 & $\mathrm{C}$ & SE & Persea odoratissima \\
\hline 22 & 2110 & 45 & B & E & Rhododendron arboreum \\
\hline
\end{tabular}

Abbreviations used: $\mathrm{SR}=$ Site representation; $\mathrm{A}=$ Dry habitat; $\mathrm{B}=$ Moist habitat; $\mathrm{C}=$ Riverine habitat; $\mathrm{D}=$ Degraded habitat; and $\mathrm{E}=$ Bouldary habitat; $\mathrm{E}=$ East; $\mathrm{N}=$ North; $\mathrm{W}=$ West; $\mathrm{S}=$ South; $\mathrm{NE}=$ North east; $\mathrm{SE}=$ South east; NW=North west; $\mathrm{SW}=$ South west; and $\mathrm{SE}=$ South east 
Table 2. Community types, distribution and major tree associates

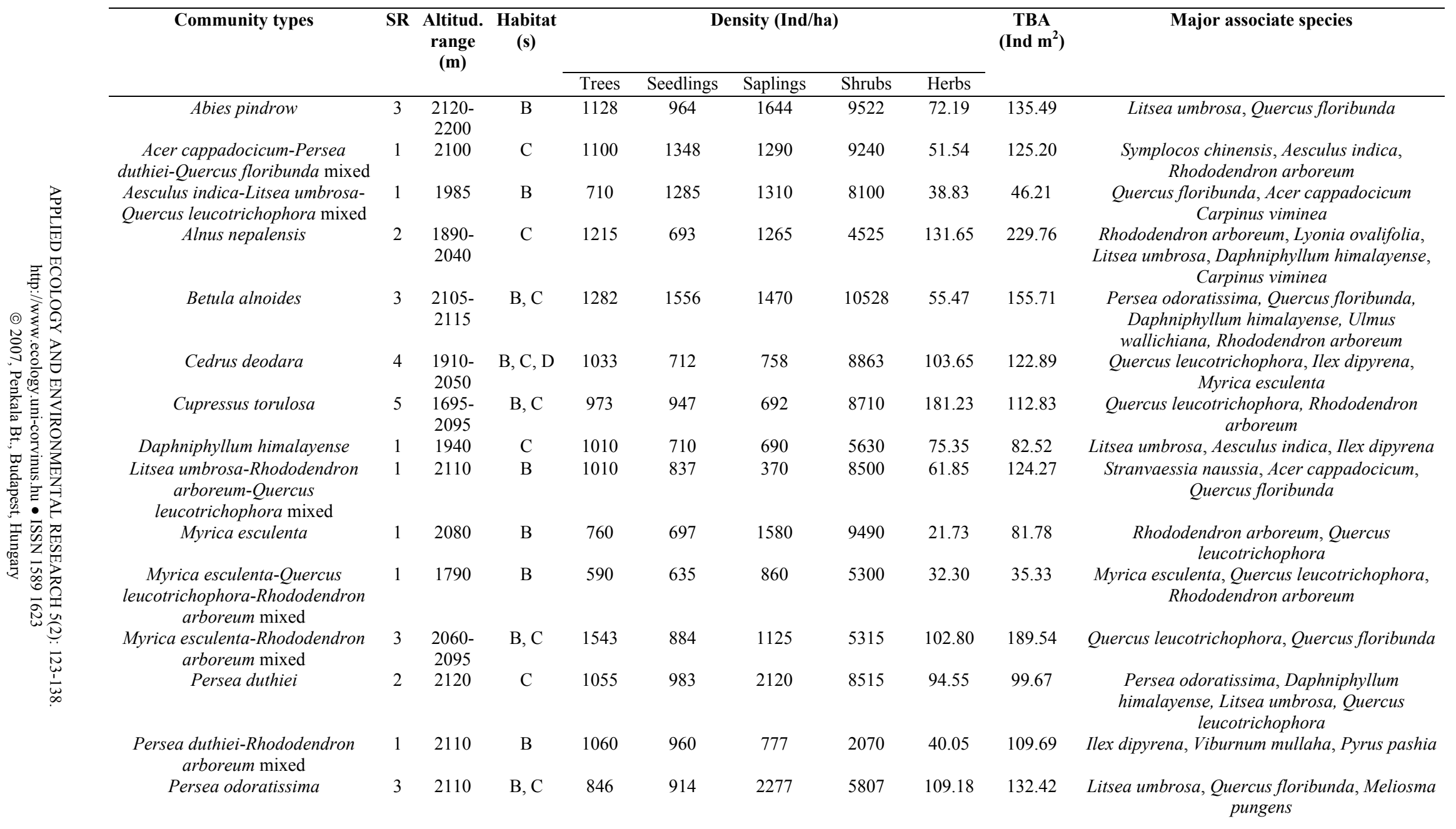




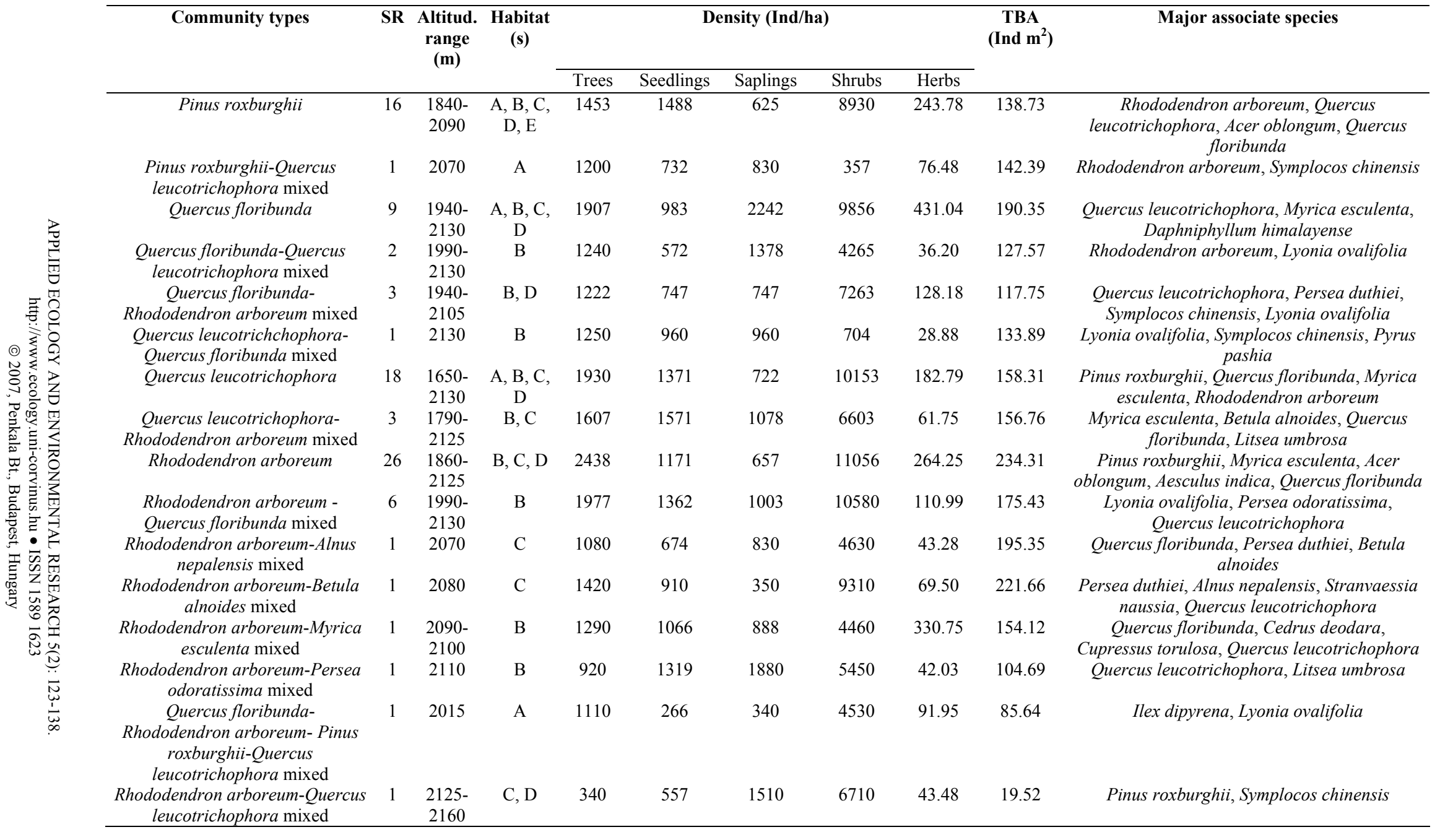

Abbreviations used: $\mathrm{SR}=$ Site representation; A= Dry habitat; B= Moist habitat; C= Riverine habitat; D= Degraded habitat; and E= Bouldary habitat 


\section{Vegetation composition}

\section{Species richness}

In all, 289 species ( 37 trees; 37 shrubs; and 215 herbs) were recorded. Richness of trees ranged from 3-27; shrubs from 8-36, herbs from 17-145, seedlings from 3-22, and saplings from 2-21. The values for trees were higher than the earlier reported values $[54,20,22]$ but comparable to the values reported by $[36,38,39]$ from high altitude areas, and also comparable to the sub-tropical and temperate regions (i.e., 9-28) [14, 33]. For shrubs, the values were slightly higher than earlier records, (4-22) from subtropical and temperate forests $[22,23,15]$. For herb layer, the values were higher than previous records [54]. The high richness of trees and shrubs may be due to diverse habitats and suitable edaphic and climatic factors supporting growth and survival of the species.

\section{Richness of native and endemic species}

Of the total 289 species, 206 species were native to the Himalayan Region; 83 species were non-natives; 117 species were near endemic; and only two species i.e., Goldfussia dalhoussiana, and Onychium fragile were endemic. Of the natives, 29 species were trees, 26 species were shrubs and 151 species were herbs. The high percentage of native species in the area may be due to unique topography, inaccessibility and distance from road heads.

Among all the communities, Rhododendron arboreum community supports maximum, native and endemic species, followed by Quercus leucotrichophora, Quercus leucotrichophora, Quercus floribunda, Rhododendron arboreum-Quercus floribunda mixed, Pinus roxburghii, Quercus floribunda-Rhododendron arboreum, Cupressus torulosa, Betula alnoides, Persea duthiei, Cedrus deodara, Myrica esculenta-Rhododendron arboreum mixed, and Alnus nepalensis, communities (Table 3).

Table 3. Richness of economically important, native, endemic, near endemic and rare endangered species in prioritized communities

\begin{tabular}{|c|c|c|c|c|c|}
\hline \multirow[t]{2}{*}{ Community types } & \multicolumn{5}{|c|}{ No. of Species } \\
\hline & Native & $\begin{array}{l}\text { Economically } \\
\text { important }\end{array}$ & Endemic & $\begin{array}{c}\text { Near } \\
\text { endemic }\end{array}$ & $\begin{array}{c}\text { Rare } \\
\text { endangered }\end{array}$ \\
\hline Rhododendron arboreum & 119 & 127 & 2 & 33 & 6 \\
\hline Quercus leucotrichophora & 97 & 98 & 2 & 25 & 6 \\
\hline Quercus floribunda & 73 & 85 & 1 & 21 & 5 \\
\hline $\begin{array}{l}\text { Rhododendron arboreum- } \\
\text { Quercus floribunda mixed }\end{array}$ & 70 & 90 & 1 & 18 & 4 \\
\hline Pinus roxburghii & 67 & 59 & 1 & 16 & 3 \\
\hline $\begin{array}{l}\text { Quercus floribunda- } \\
\text { Rhododendron arboreum mixed }\end{array}$ & 55 & 104 & 1 & 14 & 5 \\
\hline Cupressus torulosa & 55 & 69 & 1 & 19 & 2 \\
\hline Betula alnoides & 51 & 67 & 1 & 20 & 2 \\
\hline Persea duthiei & 51 & 52 & 1 & 18 & 4 \\
\hline Cedrus deodara & 46 & 59 & 1 & 11 & 1 \\
\hline $\begin{array}{c}\text { Myrica esculenta-Rhododendron } \\
\text { arboreum mixed }\end{array}$ & 45 & 68 & 1 & 18 & 2 \\
\hline Alnus nepalensis & 43 & 58 & 1 & 10 & 4 \\
\hline
\end{tabular}




\section{Structural pattern}

In general, density, TBA, and IVI of trees and density of saplings, seedlings, shrubs and herbs have been presented (Table 2). The tree density in the communities ranged from 340-2438 Ind ha ${ }^{-1}$, TBA ranged from 19.52-234.31 Ind $\mathrm{m}^{2}$, sapling density from 340.00-2277.00 Ind $\mathrm{ha}^{-1}$ and seedling density from 266.00-1571.00 Ind ha ${ }^{-1}$; shrub density ranged from 357-1156 Ind ha ${ }^{-1}$ and herb density from 21.73-431.04 Ind ha $^{-1}$; Tree density and TBA were slightly higher than the earlier reported values (320-1670 Ind $\mathrm{ha}^{-1}$ and 360-1787.50 Ind ha ${ }^{-1}$ ) from low and high altitude forests of west Himalaya $[10,8,11,16,54,22,36]$. The total shrub density for MRF is lower than the reported range for the Pindari area [20], Kedarnath Wildlife Sanctuary [30] from sub-tropical and temperate zone in Kumaun Himalaya [18]. Total herb density was ranged from (21.73431.04 $\mathrm{Ind}^{-2}$ ) for MRF, which was higher than from the reported value (0.3-17.70 tiller $\mathrm{m}^{-2}$ ) [55] but lower when compared to the Pindari and NDBR [36]. This may be due to the diversity in habitats and mild climatic conditions supporting diversity of herbaceous species and also high density of trees in the MRF. A positive correlation between and the total basal area and richness $(\mathrm{r}=0.34, \mathrm{p}<0.05, \mathrm{n}=31)$ (Fig. 1) was observed. This indicates that the increase in the species number increases the total basal cover.

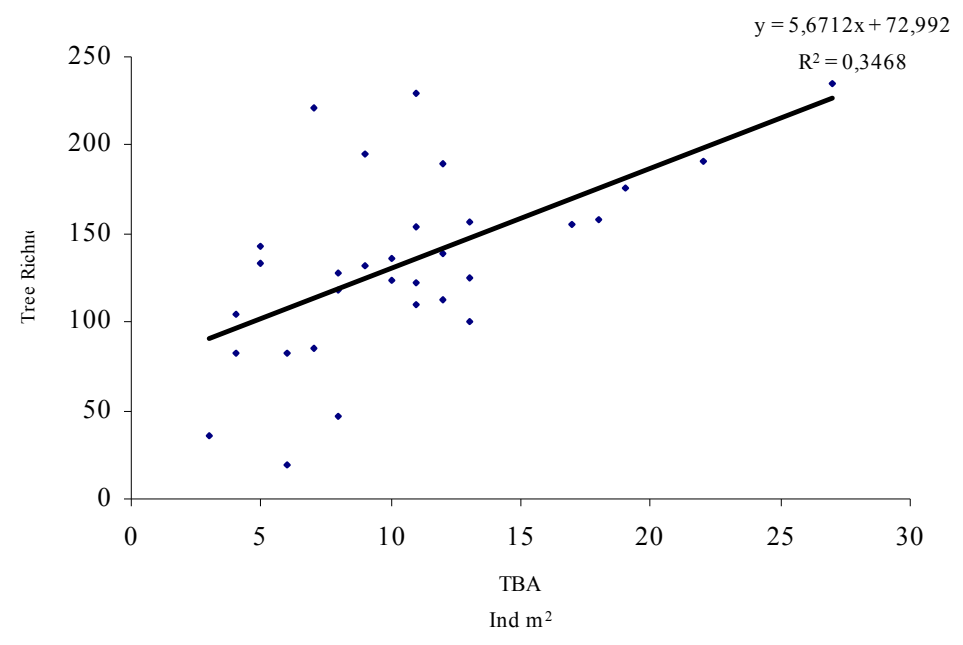

Figure 1. Correlation between Total Basal Area and Richness

\section{Species diversity ( $\left.\mathrm{H}^{\prime}\right)$}

Species diversity of trees ranged from $0.99-2.93$, seedlings from $0.86-2.65$, saplings from $0.44-2.78$, shrubs from 1.94-4.43 and herbs from 1.42-4.66. The diversity of trees was highest in the Rhododendron arboreum community (2.93), followed by Quercus floribunda (2.90), Rhododendron arboreum-Quercus floribunda mixed (2.68), and Quercus leucotrichophora (2.65), communities. The diversity of shrubs was highest in Quercus leucotrichophora community (4.43), followed by Rhododendron arboreum (3.49) and Pinus roxburghii (3.37), communities. The diversity of saplings was highest in Rhododendron arboreum (2.78), followed by Quercus floribunda (2.74), Rhododendron arboreum -Quercus floribunda mixed (2.73), communities. Diversity of 
seedlings was highest is Betula alnoides (2.65) communities, followed by Rhododendron arboreum -Quercus floribunda mixed (2.33), and Pinus roxburghii (2.21). Herb diversity was highest in Rhododendron arboreum (4.66), followed by Quercus leucotrichophora (4.48), and Pinus roxburghii (4.08). These values were comparable to the previous records for various regions of west Himalaya $[8,54,20,39$, 38].

\section{Concentration of dominance $(\mathrm{Cd})$}

Concentration of dominance of trees ranged from $0.06-0.49$, seedlings from 0.08 0.45, saplings from 0.07-0.68, shrubs from 0.03-1.00 and herbs from 0.01-0.52. Concentration of dominance of trees was highest in Myrica esculenta community (0.49), followed by Pinus roxburghii (0.46), Daphniphyllum himalayense (0.44) and Quercus leucotrichophora-Quercus floribunda mixed and Abies pindrow (0.41), communities, it was lowest in Quercus floribunda community (0.06). For shrubs, it was highest for Abies pindrow community (1.00), followed by Pinus roxburghii-Quercus leucotrichophora mixed (0.15), Persea duthiei-Rhododendron arboreum mixed (0.11), communities, and lowest for Rhododendron arboreum community (0.03). For herbs, it was highest for Rhododendron arboreum-Myrica esculenta mixed community (0.52), followed by Quercus floribunda (0.31), Persea duthiei-Rhododendron arboreum mixed (0.12) communities. These values were comparable to the previous records $[8,9]$.

\section{Socio economic and conservation values of the forest communities}

Among all the communities, Rhododendron arboreum community (208 species; 127 economically important, 119 native, 2 endemic, 33 near endemic, and 6 rare-endangered species), followed by Quercus floribunda-Rhododendron arboreum mixed (98 species, 104 economically important species, 55 native, 1 endemic, 14 near endemic, and 5 rare endangered species); Quercus leucotrichophora (179 species, 98 economically important, 97 native, 2 endemic, 25 near endemic, and 6 rare endangered species); Rhododendron arboreum-Quercus floribunda mixed (127 species, 90 economically important, 70 native, 1 endemic, 18 near endemic, and 4 rare endangered species); Quercus floribunda (150 species, 85 economically important, 73 native, 1 endemic, 21 near endemic, and 5 rare endangered species); Cupressus torulosa (116 species, 69 economically important, 55 native, 1 endemic, 19 near endemic, and 2 rare endangered species), Myrica esculenta-Rhododendron arboreum mixed ( 97 species, 68 economically important, 45 native, 1 endemic, 18 near endemic, and 2 rare endangered species), Betula alnoides ( 93 species, 67 economically important, 51 native, 1 endemic, 20 near endemic, and 2 rare endangered species), Cedrus deodara (80 species, 59 economically important, 46 native, 1 endemic, 11 near endemic, and 1 rare endangered species), Pinus roxburghii (144 species, 59 economically important, 67 native, 1 endemic, 16 near endemic, and 3 rare-endangered species); Alnus nepalensis (77 species, 58 economically important, 43 native, 1 endemic, 10 near endemic, and 4 rare endangered species); and Persea duthiei (92 species, 52 economically important, 51 native, 1 endemic, 18 near endemic, and 4 rare endangered species), communities (Table 3). This clearly indicates the high socio-economic and conservation values of these communities, hence, need prioritization for conservation. The key species of the prioritized communities are Rhododendron arboreum, Myrica esculenta, Selinium tenuifolium, Heracleum candicans, Buplerum longicaule, Berberis aristata, Sarcococa 
saligna, Viburnum cotinifolium, Quercus leucotrichophora, Q. floribunda, Hypericum oblongifolium, Salvia lanata, Artemisia nilagarica, Acorus calamus, Origanum vulgare, Melothria heterophylla, Persea duthiei, P. odoratissima, Carpinus viminea, Pyrus pashia, Michelia kisopa, Zanthoxylum armatum, Cypripedium cordigerum, Cedrus deodara, Cupressus torulosa, Taxus baccata subsp. wallichiana, Habenaria edgeworthii, H. intermedia, Prinsepia utilis, Delphinium denudatum, Skimmia laureola, Bergenia ligulata, Ulmus wallichiana, Hedychium spicatum, Pimpinella acuminata, Goldfussia dalhoussiana, Onychium fragile, and Lepisorus excavatus. The richness of economically important species in these communities indicates high anthropogenic pressure, which may lead to habitat degradation and extirpation of the species in near future. A significant positive relationship $(\mathrm{r}=0.50, \mathrm{p}<0.01, \mathrm{n}=31)$ has been found between the number of useful species and number of rare-endangered species, indicating that the use pattern of the species is directly proportional to the rarity of the species (Fig. 2.). If the rate of exploitation of the economically important species from these communities continues, there is much probability of extinction of these species from their natural habitats in near future and may lead to ecosystem imbalance. Therefore, there is an urgent need to initiate steps for the conservation of high value communities.

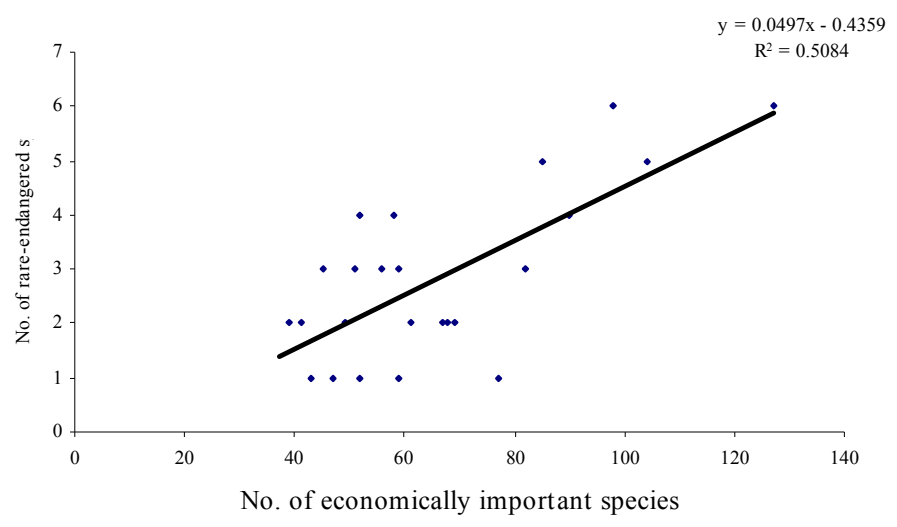

Figure 2. Correlation between Economically Important and Rare-Endangered Species

\section{Prioritization of the forest communities for conservation and management}

The conservation values of the communities are presented in (Fig. 3.) Amongst the communities, the Rhododendron arboreum community was ranked first, followed by Quercus leucotrichophora, Quercus floribunda, Rhododendron arboreum-Quercus floribunda mixed, Quercus floribunda-Rhododendron arboreum mixed, Pinus roxburghii, Cupressus torulosa, Betula alnoides, Myrica esculenta-Rhododendron arboreum mixed, Persea duthiei, Cedrus deodara, and Alnus nepalensis, communities. The prioritized communities represent the maximum numbers of economically important as well as native, endemic and rare-endangered species. 


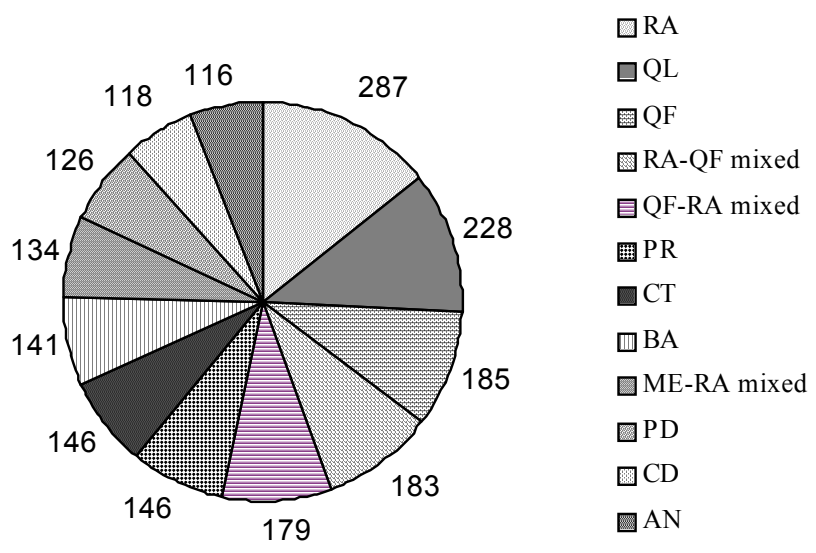

Figure 3. Prioritization of Forest communities with their Conservation Values

$\mathrm{RA}=$ Rhododendron arboreum $; \mathrm{QL}=$ Quercus leucotrichophora $; \mathrm{QF}=\mathrm{Quercus}$ floribunda; $\mathrm{RA}-\mathrm{QF}=$ Rhododendron arboreumQuercus floribunda mixed; $\mathrm{QF}-\mathrm{RA}=$ Quercus floribunda-Rhododendron arboreum mixed; $\mathrm{PR}=$ Pinus roxburghii; $\mathrm{CT}=$ Cupressus torulosa; $\mathrm{BA}=$ Betula alnoides; ME-RA mixed= Myrica esculenta-Rhododendron arboreum mixed; $\mathrm{PD}=$ Persea duthiei; $\mathrm{CD}=$ Cedrus deodara; and $\mathrm{AN}=$ Alnus nepalensis

\section{Conclusions}

The present study provides comprehensive information on site characteristic, habitats, community diversity, vegetation distribution pattern and forest composition of the species including richness of economically important, native, endemic and rareendangered species, prioritization of communities for conservation. Based on the results, it can be concluded that the area has high potential in terms of number of species and communities. The occurrence of high number of native, endemic, economically important and rare-endangered species enhance the conservation as well as socio-economic values of the MRF. The day to day need of forest resources particularly fuel and fodder species has increased the pressure on forest trees and shrubs to a great extent. Furthermore, the over-exploitation of species for fuel, fodder, medicine, food (wild edibles), and house building may lead to the extirpation of these species from the area. Therefore, there is a need to develop adequate strategy and action plan for the conservation and management of habitats, species, and communities, so that sustainable utilization of the species could be ensured.

Acknowledgements. The authors are thankful to Dr. U. Dhar, Director, G.B. Pant Institute of Himalayan Environment \& Development, Kosi-Katarmal, Almora for facilities and encouragement. One of the authors (SP) is thankful to Dr. S.C. Arya for his help during field surveys.

\section{REFERENCES}

[1] Samant, S.S. Dhar, U., Palni, L.M.S. (1998a): Medicinal Plants of Indian Himalaya: Diversity Distribution Potential Values. - Gyanodaya Prakashan, Nainital.

[2] Myers, N. (1990): The biodiversity Challenge: expanded 'hot spots' analysis. - Envir. 10(4): 243-256. 
[3] Samant, S.S., Dhar, U. (1997): Diversity, endemism and economic potential of wild edible plants of Indian Himalaya. - International Journal of Sustainable Development and World Ecology 4: 179-191.

[4] Samant, S.S. Dhar, U. Rawal, R.S. (1998b): Biodiversity status of a protected area of west Himalaya. 1-Askot Wildlife Sanctuary. - Journal of Sustainable Development and World Ecology 5: 194-203.

[5] Singh, S.P. (1998): Chronic disturbance, a principal cause of environmental degradation in developing countries. - Environmental Conservation 25 (1): 1-2.

[6] Saxena, A.K. Pandey, U., Singh, J.S. (1978): On the ecology of oak forest in Nainital Hills, Kumaun Himalaya. In: J.S. Singh and B. Gopal (eds.): Glimpses of ecology. International Scientific Publication, Jaipur, pp. 167-180.

[7] Saxena, A.K. (1979): Ecology of vegetation complex of north western catchment of river Gola. - Ph.D. Thesis submitted to Kumaun University, Nainital.

[8] Saxena, A.K., Singh, J.S. (1982a): A phytosociological analysis of woody species in forest communities of a part of Kumaun Himalaya. - Vegetatio 50: 3-22.

[9] Saxena, A.K., Singh, J.S. (1982b). Quantative profile structure of certain forests in the Kumaun Himalaya. - Proceedings of Indian National Science Academy 91: 529-549.

[10] Ralhan, P.K. Saxena, A.K., Singh, J.S. (1982). Analysis of forest vegetation at and around Nainital in Kumaun Himalaya. - Proceedings of Indian National Science Academy. B. 48: 122-138.

[11] Tewari, J.C. (1982): Vegetational analysis along altitudinal gradients around Nainital. $\mathrm{Ph} . \mathrm{D}$. Thesis submitted to Kumaun University, Nainital, India.

[12] Upreti, N. (1982): A study on phytosociology and state of regeneration of oak forest at Nainital. - Ph.D. Thesis submitted to Kumaun University Nainital. India.

[13] Saxena, A.K., Singh, J.S. (1984): The population structure of certain Himalayan forest, associations and implications concerning their future composition. - Vegetatio 50: 3-22.

[14] Upreti, N. Tewari, J.C., Singh, S.P. (1985): Oak forests of Kumaun Himalaya: composition, diversity and regeneration. - Mountain Research and Developm. 5:163-164.

[15] Tewari, J.C., Singh, S.P. (1985): Analysis of woody vegetation in a mixed oak forest of Kumaun Himalaya. - Proceedings of Indian National Science 51(3): 332-347.

[16] Kalakoti, B.S. Pangtey, Y.P.S., Saxena, A.K. (1986): Quantitative analysis of high altitude vegetation of Kumaun Himalaya. - Journal of Indian Botanical Society 65: 384396.

[17] Singh, J.S., Singh, S.P. (1987): Forest vegetation of the Himalaya. - Botanical Review, 52-53.

[18] Singh, J.S., Singh, S.P. (1986): Structure and function of the Central Himalayan oak forests. - Proceedings of Indian National Science 96: 159-189.

[19] Singh, R.S. Ralhan, P.K., Singh, S.P. (1987): Phytosociology and population structure of oak-mixed conifer forest in a part of Kumaun Himalaya. - Environment and Ecology 5(3): 475-487.

[20] Bankoti, N.S. Rawal, R.S. Samant, S.S., Pangtey, Y.P.S. (1992): Forest vegetation of inner hill ranges in Kumaun, Central Himalaya. - Tropical Ecology 33 (1): 41-53.

[21] Singh, J.S., Singh, S.P. (1992): Forest of Himalaya: Structure, Functioning and Impact of Man. - Gyanodya Prakashan, Nainital.

[22] Rawal, R.S. Bankoti, N.S., Pangtey, Y.P.S. (1994): Broad community identification of high altitude forest vegetation in Pindari catchment of Kumaun. - Proceedings of Indian National Science B. 60(6): 553- 556.

[23] Rawal. R.S., Pangtey, Y.P.S. (1994a): Distribution and structural- functional attributes of trees in the high altitude zone of central Himalaya, India. - Vegetatio 112: 29-34.

[24] Rawal, R.S., Pangtey, Y.P.S. (1994b): High altitude forests with special reference to timberline in Kumaun, central Himalaya. - In: Y.P.S. Pangtey, R.S. Rawal (eds), Altitudes of the Himalaya. Gyanodaya Prakashan, Nainital, pp. 353-399. 
[25] Rawal, R.S., Pangtey, Y.P.S. (1994c): Altitudinal zonation of high altitude forests in Kumaun, central Himalaya, India. - Indian Journal of Forestry 17(4): 332-344.

[26] Tewari, A. (1998): Tree layer analysis of three major forests forming species of Kumaun Central Himalaya. - Journal of Economic and Taxonomic Botany 11(1): 23-28.

[27] Joshi, H.C. Arya, S.C., Samant, S.S. (1999): Diversity, distribution and indigenous uses of medicinal and edible plants in a part of Nanda Devi Biosphere Reserve I. - Himalayan Biosphere Reserves (Biannual Bulletin) 1(1\&2): 49-65.

[28] Samant, S.S. Joshi, H.C., Arya, S.C. (2000a): Diversity, nativity and endemism of vascular plants in Pindari area of Nanda Devi Biosphere Reserve-II. - Himalayan Biosphere Reserves 2(1\&2): 1-29.

[29] Samant, S.S. Dhar, U., Rawal, R.S. (2000b): Assessment of fuel resource diversity and utilization patterns in Askot Wildlife Sanctuary in Kumaun Himalaya, India for conservation and management. - Environmental Conservation 27(1): 5-13.

[30] Rawat, G.S. Sathyakumar, S., Prasad, S.N. (1999): Plant species diversity and community structure in the outer fringes of Kedarnath Wildlife Sanctuary, Western Himalaya: Conservation implications. - Ind. For. 125(9): 873-882.

[31] Rawat, G.S. Kala, C.P., Uniyal, V.K. (2001): Plant species diversity and community composition in the Valley of Flowers, National Parks, Western Himalaya. - In: P.C. Pande and S.S. Samant (eds.), Plant Diversity of the Himalaya. Gyanodaya Prakashan, Nainital. pp. 277-290.

[32] Bankoti, N.S. Tewari, L.M. (2001): Analysis of forest vegetation at and around SoniBinsar area in Kumaun Himalaya. - In: P.C. Pande, and S.S. Samant (eds), Plant diversity of the Himalaya, Gyanodaya Prakashan, Nainital pp. 363-376.

[33] Rawat, R.S. (2001): Phytosociological studies of woody vegetation along an altitudinal gradient in a montane forest of Garhwal Himalaya. - Indian Journal of Forestry, 24(4): 419-426.

[34] Pant, S. (2005): Plant Diversity and Ethnobotany of Mornaula Reserve Forest in Kumaun, West Himalaya. - Ph.D. Thesis submitted to Kumaun University, Nainital. India.

[35] Dhar, U. Rawal, R.S., Samant, S.S. (1997): Structural diversity and representativeness of forest vegetation in a protected area of Kumaun Himalaya, India: implications for conservation. - Biodiversity and Conservation 6: $1045-1062$.

[36] Samant, S.S. Joshi, H.C. Arya, S.C., Pant, S. (2002): Studies on the structure, composition and changes of the vegetation in Nanda Devi Biosphere Reserve of West Himalaya. - Final Technical Report submitted to Ministry of Environment and Forests, New Delhi.

[37] Joshi, H.C., Samant, S.S. (2004). Assessment of forest vegetation and prioritization of communities for conservation in a part of Nanda Devi Biosphere Reserve, West Himalaya, India I. - International Journal of Sustainable Development and World Ecology 11: 326-336.

[38] Samant, S.S., Joshi, H.C. (2005): Plant diversity and conservation status of Nanda Devi National Park and comparisons with highland National Parks of Indian Himalayan Region. - International Journal of Biodiversity Science and Management 1: 65-73.

[39] Kersaw, K.A. (1973): Quantitative and dynamic plant ecology. - Second edition. Edward Arnold Limited, London.

[40] Curtis, J.T., Intosh, Mc. (1950): The interrelation of certain analytic and phytosociological characters. - Ecology 31: 434-455.

[41] Greig-Smith, P. (1957): Quantitative plant ecology. - Academic Press, New York.

[42] Mueller-Dombois, D., Ellenberge, H. (1974): Aims and methods of vegetation ecology. John Willey and Sons, New York.

[43] Osmaston, A.E. (1927): A Forest Flora for Kumaun. - International Book Distributors, Dehra Dun (Reprinted 1978).

[44] Naithani, B.D. (1984 \& 85): Flora of Chamoli, Vol I - II. Botanical Survey of India Howrah, New Delhi. 
[45] Samant, S.S. (1987): Flora of Central and South Eastern Parts of Pithoragarh District. Vol. I \& II. - Ph. D. Thesis submitted to Kumaun University, Nainital.

[46] Shannon, C.E., Wiener, W. (1963): The Mathematical Theory of Communication. University of Illinois Press, Urbana.

[47] Simpson, E.H. (1949): Measurement of diversity. - Nature, 163-688.

[48] Anonymous (1883): Index Kewensis Plantarum Phanerogamarum Vol. 1-2 (1883-1885) and 15 Suppl. (1886-1970). - Clarendron Press, Oxford.

[49] Samant, S.S. (1999): Diversity, nativity and endemism of vascular plants in a part of Nanda Devi Biosphere Reserve in west Himalaya I. - Himalayan Biosphere Reserves (Biannual Bulletin) 1(1\&2): 1-28.

[50] Dhar, U., Samant, S.S. (1993). Endemic diversity of Indian Himalaya I. Ranunculaceae and II. Paeoniaceae. - Journal of Biogeography 20: 659-668.

[51] Samant, S.S. Dhar, U. Rawal, R.S. (1996): Natural resource use by some natives within Nanda Devi Biosphere Reserve in west Himalaya. - Ethnobotany 8: 40-50.

[52] Samant, S.S., Pal, M. (2003): Diversity and conservation status of medicinal plants in Uttaranchal State. - Indian Forester 129(9): 1090-1108.

[53] Adhikari, B.S. Rikhari, H.C. Rawat, Y.S., Singh, S.P. (1991): High altitude forest: composition, diversity and profile structure in a part of Kumaun Himalaya. - Tropical Ecology 32 (1): 86-97.

[54] Chandra, R. Upadhyaya, V.P., Bargali, S.S. (1989): Analysis of herbaceous vegetation under Oak and Pine Forests along an altitudinal gradient in central Himalaya. Environment and Ecology 7(3): 521-525. 